\title{
The role of psycho-education in improving outcome at a general hospital psychiatry clinic in Uganda
}

\author{
E Prost ${ }^{1}$, S Musisi ${ }^{2}$, ES Okello², WM Hopman ${ }^{3}$ \\ 1Department of Psychiatry, Queen's University, Kingston, Canada \\ 2Department of Psychiatry, Makerere University, Kampala, Uganda \\ ${ }^{3}$ Clinical Research Centre, Kingston General Hospital, and Department of Community Health and Epidemiology, Queen's University, \\ Kingston, Canada.
}

\begin{abstract}
Objective: While psychoeducation has been shown to positively affect outcomes in psychiatric disorders, its utility has been little studied in developing countries. The current study sought to examine the role of psychoeducation at a general psychiatric outpatient clinic in Kampala, Uganda in improving clinic attendance, treatment adherence, and clinical outcomes. Method: A prospective casecontrol study using a quasi-experimental design was conducted in 117 patients suffering various psychiatric disorders. Participants were recruited for two months and then followed for a further three months after recruitment ended. Participants in the intervention group received formalized psychoeducation sessions at each clinic visit in addition to the usual psychiatric evaluation and care. Participants in the control group received the usual clinical care. Measured outcomes were knowledge of mental illness, compliance with medications and follow-up, and Clinical Global Impression (CGI). Results: The groups did not differ with respect to sociodemographic characteristics or attendance at scheduled follow-up visits. Both groups significantly improved on the CGI, but with no significant difference between the groups. However, the intervention group was more likely to adhere to medication, and their knowledge of mental illness was significantly higher at follow-up. Conclusion: These data suggest that psychoeducation is a beneficial mental health intervention in a developing country that may increase compliance with medication and result in greater knowledge of mental illness. However, other factors such as distance from a centralized clinic or cost of treatment may impact outcomes, including attendance at scheduled follow-up visits.
\end{abstract}

Keywords: Low and middle income countries; Outpatient treatment; Psychosocial interventions; Transcultural Psychiatry; Africa; Psychoeducation

Received: 10-05-2011

Accepted: 18-06-2012

doi: http://dx.doi.org/10.4314/ajpsy.v16i4.35

\section{Introduction}

Psychoeducation usually refers to the imparting of knowledge about mental illness to a patient and his or her family. It is often informal and includes discussions of etiology, natural history, and treatments, as well as ways to maintain health such as being alert to early signs of deterioration, enhancing medication compliance, and avoiding substances.

Psychoeducation has been adopted as an integral part of many specialized and general psychiatry clinics and is increasingly being recognized as a formalized form of treatment in itself with accumulating evidence for its positive influence on a number of outcome measures ${ }^{1,2}$ Psychoeducation programmes for specific illnesses have shown benefit. For example, family education in Schizophrenia improved patients' social functioning ${ }^{3}$, and relapse rates decreased in Schizophrenia with family and 
patient psychoeducation. ${ }^{4}$ In bipolar disorder, the number of mood episodes, hospitalization rates, and the time between mood episodes were all affected positively when a psychoeducation programme was implemented. ${ }^{5}$ Evidence showing benefit for patients with other diagnoses - obsessive compulsive disorder, major depressive disorder, and post-traumatic stress disorder is accumulating. ${ }^{6}$

Uganda, a country of 28 million in East Africa, has a psychiatrist: population ratio of $1: 1,000,000 .{ }^{7}$ It is therefore necessary to use the psychiatrists' expertise to maximum effect while continuing to train other mental health professionals in evidence-based care and exploring creative ways to deliver it. Because of the limited resources for health and especially for mental health, studies and programmes that require minimal equipment, laboratories, and infrastructure are advantageous. Training for those delivering psychoeducation to patients and families costs little, and the number of patients and familymembers each trainee can then reach is large. Moreover, because such programmes require little technology, electronic resources, or buildings, they can be continued and expanded, even to rural areas, once implemented. Effective psychoeducation can be delivered out-of-doors or while patients and families wait for appointments, and can be adapted to different cultures and settings.

This last point - different cultures and settings - merits attention. Psychoeducation, by its very nature, deals with differing views of mental illness and thus engages culture and belief patterns. For example, if a culture views the symptoms of mental illness as a curse, or brought by fate and thus unchangeable, or as a result of sins committed, these may all need to be addressed in the education. Simply imposing a completely biologically-based model of mental illness during the education may be neither accurate nor helpful. These issues need to be addressed when designing psychoeducation for a setting in the developing world.

While most of the psychoeducation literature deals with developed countries, a recent intervention was studied in Nigeria where psychiatric inpatients with psychosis received psychoeducation sessions. The intervention proved effective for subsequent scheduled outpatient appointment compliance. ${ }^{8}$ The aim of the present study was to conduct a prospective case-control trial of formalized psychoeducation in outpatients at a general hospital psychiatry clinic in Kampala, Uganda. Unlike the Nigerian study, we recruited outpatients of all diagnoses, and measured, not only compliance with scheduled follow-up appointments, but also knowledge of mental illness, adherence to treatment, and general mental status improvement. It was hypothesized that patients receiving the psychoeducation would improve on the above measures.

\section{Method}

Subjects

The 117 participants were adults 18 years of age and older (or turning 18 during the study duration). The study rationale was to evaluate the effect of psychoeducation in a busy working clinic under real-life conditions; therefore, it was not a randomized controlled trial. Rather, the research was designed to evaluate the intervention itself as a relatively inexpensive psychoeducation programme which might be implemented in other settings in developing countries, and to do so with a minimum of disruption to the functioning clinic. We, therefore, employed a prospective case-control study using a quasi-experimental design. The control and intervention groups were recruited on alternate days from all new adult patients presenting to the clinic. The control group consisted of 53 participants and the intervention group 64.

The patients were enrolled at the S. B. Bosa Mental Health Unit at Mulago Hospital in Kampala, Uganda. It is a general psychiatric outpatient clinic at the country's main general national referral hospital. New patients present daily at the clinic from the hospital's own casualty department, community medical clinics, district hospitals, or of their own accord. All new patients who were to turn at least 18 years-old during the study were eligible and invited to participate. One patient spoke a language none of the clinic staff knew and was, therefore, ineligible, while another patient withdrew his consent later during the initial visit and was not included in the analysis. All other participants who signed informed consent were included in the statistical analysis. Because one of the outcome measures was compliance with scheduled follow-up appointments, failure to continue attending at the clinic was not considered withdrawal from the study.

\section{Study Procedures}

Before commencement of the study, study personnel were trained in a formalized 3-session psychoeducation intervention. Each session was designed to be approximately 45 minutes long. The first session addressed basic characteristics of mental disorders mood disorders, psychosis, seizure disorders - including precipitating and perpetuating factors and signs of relapse. The second session focused on cultural aspects of mental illness including demystifying patients' and families' beliefs about causes of mental illness and common attitudes including stigma. The final session addressed treatments including medication options, the importance of compliance, how to recognize side effects, and duration of use, as well as stressing the deleterious effects of substances on mental health.

New patients turning at least 18 years-old during the study duration who came to the clinic over a 2-month period were identified. After the study had been fully explained and informed consent obtained, their demographic data - age, sex, marital status, religion, tribe, diagnosis, medications, distance traveled to clinic, whether they attended with family members, education level attained - were recorded. At the initial visit, the Knowledge of Mental Illness Questionnaire was administered as well as the Clinical Global Impressions scale, the latter by a psychologist blinded to the patient's inclusion in the intervention or control group.

The sessions were administered to the intervention group participants and their families in the outpatient 
clinic while they waited to see the clinicians for their appointments. They were administered in small groups or individually depending on the number of patients each day. The sessions were given on a two-weekly rotating basis: for the first two weeks, session 1 was given, for the third and fourth weeks, session 2 was given, and so on. The sessions were designed so that a patient would benefit if he or she received them in any order. The control group did not receive any psychoeducation sessions.

For the first two months of the study, patients for both groups were enrolled and the intervention group patients received the psychoeducation sessions being given on each day they attended the clinic. After 2 months, enrolment of new patients stopped but the psychoeducation sessions continued so that the enrolled patients in the intervention group continued to receive the sessions each time they attended for the full five months of the study. Ideally, all intervention group patients who started receiving psychoeducation (that is, who attended their first psychoeducation session) would have completed all three sessions, thus allowing the intervention as a whole to be correlated with the outcome measures. However, even if patients completed only part of the intervention (that is, attend 1 or 2 sessions), they were included in the analysis. The clinicians evaluating and treating all patients in both groups were not study staff and were blinded to the group inclusion of their patients.

\section{Outcome Measures}

Scheduling of follow-up appointments was determined by the blinded clinicians without consideration of the study procedures. Study personnel simply recorded whether the participant then arrived on the scheduled date. If the patient failed to do so within one week of the scheduled follow-up, he or she was recorded as a no show. Patients' knowledge of mental illness was measured at each visit using the Knowledge About Depression and Mania Inventory ${ }^{9}$, modified to apply to the cultural milieu in Uganda. Depression and Mania were the chosen disorders for inclusion in this measurement as they were the most common ones at the S.B. Bosa Mental Health Unit at Mulago hospital. General improvement in patients' mental status was measured at each visit using the Clinical Global Impressions (CGI) administered by a blinded psychologist. Finally, adherence to prescribed medications was determined as a yes/no by chart reviews of each participant at the end of the study. A participant was deemed a "no" if, at any point in the chart, there was evidence of non-compliance.

\section{Statistical Analysis}

Data were entered into an Excel spreadsheet designed for the study, and imported into SPSS (version 17 for Windows) for statistical analysis. Following a descriptive analysis (frequencies and percentages for categorical data; means and medians for continuous data), the two groups were compared using chi-square tests (Pearson or Fisher's Exact as appropriate) and independent samples t-tests. Paired samples t-tests were used to assess change in knowledge of mental illness and CGI both overall and within the two groups. Chi-square tests were also used to examine whether changes in CGI differed depending on the level of CGI at baseline.

\section{Ethics}

Approval was granted by the Makerere University, Faculty of Health Sciences, review board. Approved consent forms were explained and signed by all participants.

\section{Results}

\section{Adverse Effects}

None of the participants reported any adverse sideeffects to the psychoeducation intervention, including no concerns regarding longer waits at the clinic as most of the sessions were administered while patients were waiting, given the clinic's usual functioning routine.

\section{Outcomes}

Group characteristics are presented in Table I. The groups did not differ significantly with respect to age, gender, psychiatric and medical co-morbidities, education level, whether or not they had family accompanying them, or distance travelled to the clinic. This latter measure showed a trend towards urban dwellers in the control group ( $\mathrm{p}=0.095)$ but the distance travelled did not differ significantly (mean 23.8 for control versus 25.2 for intervention, $p=0.88$ ). The groups did differ with respect to substance abuse, with the intervention group having 9 with this diagnosis and the control group $0(\mathrm{p}=0.004)$.

Results of the measured outcomes are presented in Tables II and III. Overall, knowledge of mental illness showed an improvement in mean scores, from 7.96 to 8.78, which fell just short of statistical significance $(\mathrm{p}=0.068)$. However, when examined within the treatment groups, the control group saw less improvement (mean 7.71 to $7.90, p=0.770$ ) than the intervention group, which saw statistically significant changes (mean 8.16 to 9.52 , $\mathrm{p}=0.032)$.

CGI showed a statistically significant improvement overall $(p<0.001)$, with an average change of one category. The most frequent change was from "markedly ill" to "moderately ill", but there was no evidence to suggest that the most severely ill saw the greatest improvement. Rather, the improvements were distributed relatively equally across the baseline CGI levels $(p=0.646)$. However, there was no significant effect of intervention on the change in CGI. While both groups saw significant improvement, the magnitude of improvement did not differ between the groups ( $p=0.89)$.

Adherence to medication did differ between the groups, with the control group considered compliant in $80.6 \%$ of cases, while the intervention group was compliant in $97.0 \%$ of cases, $\mathrm{p}=0.049$. However, the number of appointments attended did not differ between the two groups. The intervention group attended an average of 2.2 appointments while the control group attended an average of 2.5 appointments, $p=0.252$. Insufficient volume in each of the disorders, and loss to follow-up, prohibited useful diagnosis-specific analyses. 


\begin{tabular}{|c|c|c|c|}
\hline \multicolumn{4}{|c|}{ Table I: Baseline Characteristics by Group } \\
\hline Characteristic & $\begin{array}{l}\text { Control Group } \\
(n=53)\end{array}$ & $\begin{array}{l}\text { Intervention Group } \\
(n=64)\end{array}$ & $p$-value \\
\hline & Mean $(S D)$ & Mean (SD) & \\
\hline Age (range $17-85$ years) & $31.1(12.5)$ & $30.9(13.9)$ & 0.94 \\
\hline \multirow[t]{2}{*}{ Distance (range 0-400 km) } & $23.8(59.4)$ & $25.2(36.8)$ & 0.88 \\
\hline & Frequency (\%) & Frequency (\%) & \\
\hline $\begin{array}{l}\text { Male } \\
\text { Urban Residence }\end{array}$ & $\begin{array}{l}28(52.8) \\
40(76.9)\end{array}$ & $\begin{array}{l}37(57.8) \\
40(62.5)\end{array}$ & $\begin{array}{l}0.59 \\
0.10\end{array}$ \\
\hline $\begin{array}{l}\text { Education } \\
\text { S Primary } \\
\text { Some / Completed Senior } 6 \\
\text { Technical / University }\end{array}$ & $\begin{array}{l}13(24.5) \\
26(49.1) \\
13(24.5)\end{array}$ & $\begin{array}{l}16(25.0) \\
29(45.3) \\
15(23.4)\end{array}$ & 0.24 \\
\hline $\begin{array}{l}\text { Comorbidities } \\
\text { Hypertension } \\
\text { HIV Positive } \\
\text { Head Injury }\end{array}$ & $\begin{array}{l}3(5.7) \\
6(11.3) \\
2(3.8)\end{array}$ & $\begin{array}{l}1(1.6) \\
8(12.5) \\
4(6.3)\end{array}$ & $\begin{array}{l}0.32 \\
0.86 \\
0.69\end{array}$ \\
\hline $\begin{array}{l}\text { Major Psychiatric Diagnoses } \\
\text { Schizophrenia } \\
\text { Bipolar Disorder } \\
\text { Major Depression } \\
\text { Post Traumatic Stress Disorder } \\
\text { Other Anxiety Disorder } \\
\text { Seizure Disorder } \\
\text { Substance Abuse } \\
\text { Other Disorders }\end{array}$ & $\begin{array}{l}3(5.7) \\
8(15.1) \\
16(30.2) \\
4(7.5) \\
4(7.5) \\
10(18.9) \\
0(0.0) \\
13(24.5)\end{array}$ & $\begin{array}{l}7(11.5) \\
8(12.5) \\
13(20.3) \\
1(1.6) \\
2(3.1) \\
11(17.2) \\
9(14.1) \\
17(26.6)\end{array}$ & $\begin{array}{l}0.32 \\
0.67 \\
0.20 \\
0.17 \\
0.41 \\
0.79 \\
0.004 \\
0.91\end{array}$ \\
\hline
\end{tabular}

\section{Loss to Follow-up}

Due to the fact that a large number of participants attended the clinic only once (44\%), the baseline characteristics of those who were lost to follow-up were compared to those who attended at least one follow-up session. Results suggest that there were few differences between the groups. There were no significant differences in age $(p=0.68)$, distance $(\mathrm{p}=0.70)$, baseline CGI ( $\mathrm{p}=0.87)$, or baseline knowledge $(p=0.68)$. Those lost to follow-up were equally distributed between the control and intervention group ( $\mathrm{p}=0.96)$, and did not differ by sex $(p=0.61)$. Medical conditions did not differ significantly, and only one of the eight major psychiatric disorders differed, in that those with bipolar disorder were somewhat less likely to have follow-up data $(p=0.031)$. The similarity between the two groups suggests that the data for those with follow-up would be reasonably representative of the larger sample.

\section{Discussion}

The study groups did not differ with respect to sociodemographic characteristics. However, the results of this study in a busy outpatient psychiatry clinic in Uganda showed that a formalized psychoeducation intervention was positively associated with improved patient knowledge of mental illness and compliance with prescribed medications. There were no measurable significant differences in attendance at scheduled followup appointments or improvement on the Clinical Global Impressions scale - the two validated outcome measures in this study.

\section{Attendance at Scheduled Follow-ups}

Most disorders treated as mental illnesses in Uganda such as schizophrenia, bipolar disorder, epilepsy, etc are chronic. Treatment is often life-long and compliance with medication regimes essential for better outcomes. Therefore, attendance at scheduled follow-up appointments for mental status checks, medication reviews, and support would seem essential. Also, diagnosis does not always occur at the first visit: psychotic and mood disorders evolve and often diagnosis becomes clearer only after evaluating a patient longitudinally. Therefore, again, attendance at follow-up appointments seems essential. However, this outcome 
Table II: Knowledge of mental illness and CGI scores at baseline and at 3 month follow-up

\begin{tabular}{|c|c|c|c|}
\hline Characteristic & Control Group & Intervention Group & $p$-value \\
\hline & Mean (SD) & Mean (SD) & \\
\hline Baseline Knowledge of Mental IIIness (1-20) & $\begin{array}{l}N=53 \\
7.9(3.5)\end{array}$ & $\begin{array}{l}N=61 \\
8.3(3.0)\end{array}$ & 0.51 \\
\hline Follow-up Knowledge of Mental Illness (1-20) & $\begin{array}{l}N=21 \\
7.9(3.9)\end{array}$ & $\begin{array}{l}N=27 \\
9.2(3.7)\end{array}$ & 0.23 \\
\hline Within-group change & $\begin{array}{l}N=21 \\
0.19(2.9) \\
p=0.77\end{array}$ & $\begin{array}{l}N=25 \\
1.36(3.0) \\
p=0.032\end{array}$ & \\
\hline & Frequency (\%) & Frequency (\%) & \\
\hline $\begin{array}{l}\text { Baseline Clinical Global Impression } \\
\text { Borderline/Mildly III } \\
\text { Moderately III } \\
\text { Markedly III } \\
\text { Severely/Extremely III }\end{array}$ & $\begin{array}{l}N=53 \\
4(7.5) \\
17(32.1) \\
17(32.1) \\
15(28.3)\end{array}$ & $\begin{array}{l}\mathrm{N}=62 \\
2(3.1) \\
16(25.0) \\
25(39.1) \\
19(29.7)\end{array}$ & 0.24 \\
\hline $\begin{array}{l}\text { Follow-up Clinical Global Impression } \\
\text { Borderline/Mildly III } \\
\text { Moderately III } \\
\text { Markedly III } \\
\text { Severely/Extremely III }\end{array}$ & $\begin{array}{l}\mathrm{N}=21 \\
7(33.3) \\
12(57.1) \\
2(9.5) \\
0(0.0)\end{array}$ & $\begin{array}{l}\mathrm{N}=27 \\
6(22.2) \\
15(55.6) \\
4(14.8) \\
2(7.4)\end{array}$ & 0.46 \\
\hline
\end{tabular}

$\mathrm{SD}=$ standard deviation

P-values are based on independent samples t-tests (continuous data) or chi-square tests (categorical data), using Fisher's Exact test.

measure, positively affected by inpatient psychoeducation in Agara's Nigerian study, was not so affected in our study. A large number of our study participants from both groups only attended the clinic once (44\%). There may be several reasons for this. Perhaps psychoeducation could not change this attendance pattern in the face of the distances necessary to travel to the clinic and the cost of transportation, as, overall, $31 \%$ of participants lived in rural areas. Moreover, even travel within the capital city itself was often time-consuming and relatively costly.

Also, by the time many patients had reached our urban hospital psychiatry clinic, they had often tried other closer routes to care such as traditional healers or local clinics and, therefore, tended to be quite ill at

\section{Table III: Attendance rates at follow up and adherence to medication regimens}

\begin{tabular}{|c|c|c|c|}
\hline Characteristic & $\begin{array}{l}\text { Control Group } \\
N=53\end{array}$ & $\begin{array}{l}\text { Intervention Group } \\
N=64\end{array}$ & $p$-value \\
\hline & Mean (SD) & Mean (SD) & \\
\hline $\begin{array}{l}\text { Number of Workshops Attended* } \\
\text { Basic characteristics } \\
\text { Cultural aspects } \\
\text { Treatment }\end{array}$ & $\begin{array}{l}n / a \\
n / a \\
n / a\end{array}$ & $\begin{array}{l}1.3(0.5) \\
1.5(0.7) \\
1.2(0.4)\end{array}$ & $\begin{array}{l}n / a \\
n / a \\
n / a\end{array}$ \\
\hline \multirow[t]{2}{*}{ Number of Appointments Attended } & $2.5(1.7)$ & $2.2(1.5)$ & 0.31 \\
\hline & Frequency (\%) & Frequency (\%) & \\
\hline Adherence to Medication at follow-up & $\begin{array}{l}N=31 \\
25(80.6)\end{array}$ & $\begin{array}{l}\mathrm{N}=28 \\
32(97.0)\end{array}$ & 0.049 \\
\hline
\end{tabular}

* Patients were encouraged to attend the workshops even if they had previously participated in a workshop for that topic.

P-values are based on the Mann-Whitney U (number of appointments was not normally distributed) and the chi-square test (Fisher's Exact) 
presentation to us (the mean CGI score at initial assessment was 4.9 or "markedly ill"). While compliance with medication was significantly higher in the intervention group, it was generally quite high in both groups. Because so many participants only attended once, data on compliance with medications at follow-up was incomplete; however, of those who attended more than once, 89.1\% overall adhered to medication. If this severity of illness is coupled with the cultural tendency to follow the advice of medical authorities in taking medicines, perhaps patients decided simply to continue with the treatment initially prescribed, and which was working, rather than undergoing the expense and inconvenience of a return visit. We do not have data to prove this, but it may explain our results, which do show that the majority of patients in either group who did return for follow-up had improved significantly with treatment - from the mean CGI score at initial assessment of 4.9 ("markedly ill") to the mean at final follow-up of 3.9 ("moderately ill"). Similar improvements may have been present in those patients who failed to return, and even explain their failure to follow-up.

What is clear is that $44 \%$ of the participants only attended the clinic once during the study duration, and that the psychoeducation intervention did not improve attendance. Other factors may have influenced this loss to follow-up

\section{Overall Improvement}

It is heartening to find from this study that significant improvements in those suffering from serious mental illnesses were, and are being, achieved at our clinic, with or without our psychoeducation intervention: both groups improved significantly on the CGI. This likely speaks less to the severity of illness at initial presentation and more to the nature of the illnesses presenting, as well as to the skill and dedication of the clinical staff. The most frequent change in CGI score was from "markedly ill" to "moderately ill". However, the most severely ill did not necessarily show the greatest improvement; instead, improvement averaging one category was relatively evenly distributed across the baseline CGI levels. The main treatment modality administered by the clinic staff was medication rather than psychotherapy (less than 5\% of study participants overall did not receive medication), and it was after taking medication that most patients who did return for follow-up had significantly improved.

Psychiatric illness that responds markedly to medication is not necessarily more severe, but it is likely of a quality often called "biological" or "endogenous" or other nebulous yet useful terms. Many patients presented with psychosis, mood disorders with neurovegetative signs, or seizure disorders. These disorders are considered by many to have a largely biological basis, hence the good response to biological interventions.

The clinic's supply of psychiatric medications for free distribution to these patients was mostly restricted to older less-expensive drugs like tricyclic antidepressants and typical antipsychotics. While these worked well for many patients, the supply was inconsistent. Given our findings, future interventions might target a consistent supply of the most useful and tolerable medications as well as the provision of psychoeducation sessions.

Our psychoeducation intervention, while addressing cultural differences and local beliefs about causes of mental disorders, mainly instilled a Western biologicallybased explanation of mental disorders, thus attempting to de-mystify and possibly de-stigmatize mental illness. Our results regarding medication use confirm that many of our patients did indeed suffer from disorders that responded to biological treatments, but psychoeducation using this model did not necessarily bring consistently positive or significant results as we had expected in regard to scheduled follow-up attendance.

\section{Substance Abuse}

As above (and in Table I), the control and intervention groups did not differ significantly with respect to demographic characteristics. They were also similar in their numbers of each major psychiatric diagnosis. The only statistically significant difference between groups pertained to substance abuse, where the control group had 0 participants with this diagnosis and the intervention group 9 .

When the data regarding these participants were examined, however, the presence of these participants in the intervention group did not significantly influence the results. These 9 participants were just as likely to attend the psychoeducation workshops as patients with other diagnoses, and just as likely to attend follow-up appointments in general. If anything, they were slightly more likely to adhere to medication than participants with other diagnoses. Because those who abused substances were all in the intervention group, it is impossible to tell from the data if patients who abused substances were more likely to adhere to medications, or if patients who abused substances and received psychoeducation were more likely to adhere to medications.

\section{Limitations of Measuring Tools}

Only the less robust outcome measures - medication compliance and knowledge of mental illness - showed statistically significant benefits in the intervention group. While the raters of medication compliance were blinded to the group-membership of each patient whose chart they were reviewing, and while they considered a participant a "no" if at any time in the chart there was evidence of medication non-compliance, it may have been the case that some participants did not take their medications as prescribed and yet did not have written evidence of this in their charts. Also, while the knowledge of mental illness questionnaire resulted in a quantifiable score each time a participant completed it, the translated version used in this study has not been independently shown to be valid and reliable. Kronmueller's original German version has a published research literature for its use. However, after translation into English and Luganda, and after culturally-specific questions had been added, its properties can no longer be assumed. It is promising that both medication compliance and knowledge of mental illness improved with our intervention, but both require 
further study to discern their significance in the context of the other primary outcome measures being unaffected by the intervention.

\section{Cultural Differences}

Several models of psychoeducation could have been used. Some are narrower such as attempts to increase compliance to specific medications targeting certain disorders. For example, a pilot study in South Africa focused on psychoeducation for depression and its treatment, specifically targeting drop-out rates during SSRI treatment. ${ }^{10}$ Another recently studied model is the mutual support group in which patients' families lead the intervention by meeting in groups without the patients, the aim being to decrease family burden and increase family coping. ${ }^{11}$

We chose our model - a formalized education program aimed at all patients and their attendant families regardless of diagnosis - for several reasons. First, we wanted to insert a programme into an existing clinic, with little disruption, and minimal resources and expense. Given the pre-existing style of the clinic before our intervention, we could not separate patients by diagnosis in order to study just one or to keep the participants homogeneous. This is often an aim in the mutual support group model. ${ }^{12,13}$ Nor was this desirable. The goal was to offer a more general model of psychoeducation applicable to multiple diagnoses. Second, while some patients and families may have received information about their disorders from their clinicians during clinical visits, no formal psychoeducation existed before our intervention. Therefore, we were not building on a knowledge base or an existing programme. While mutual support groups and other models are not predicated on the participants' having preexisting knowledge of mental disorders, some models focus in detail on specific aspects of an illness and are highly specialized, building on the basics to greater degrees. Again, our model was designed to instill the basics.

The issue of cultural differences is important. As described above, the model used was biologically-based but focused on social and psychological aspects of mental illness as well - substance use, the importance of certain social stresses - and promoted the use of several "Western" models of psychotherapy as possible treatments. Cultural differences in our setting were accounted for in the design by three measures. First, all staff administering psychoeducation were local staff. When this was coupled with their flexibility to interact with patients and families case-by-case and group-bygroup within the psychoeducation framework, they could respond to cultural differences, not delivering a onedimensional model. Second, we had just one blinded (and local) psychologist administer the CGI; thus this was constant across all patients throughout the study. Third, the psychoeducation intervention focused mostly on symptoms of mental illness rather than theories of causes. However, in the end, the point was to administer a unified psychoeducation model, the content of which we believed to be accurate, and then to measure its impact within the culture of Uganda.

\section{Conclusion}

Psychoeducation remains a relatively inexpensive and easyto-apply mental health intervention in a developing country. Our positive findings have implications for the long-term management of patients with chronic mental illnesses in Africa, where beliefs in the supernatural often compromise the care of the mentally ill. However, challenges remain since other factors such as distance from a centralized clinic or cost of treatment may be important determinants of whether a patient attends for treatment at scheduled followup visits.

\section{Acknowledgement}

E.P. was supported by a research initiation grant from Queen's University.

\section{References}

1. Dowrick C, Dunn G, Ayuso-Mateos JL, Dalgard OS, Page H, Lehtinen $V$, et al. Problem solving and group psychoeducation for depression: multicentre randomized controlled trial. Outcome of Depression International Network (ODIN) Group. BMJ 2000; 321 : 1450-4.

2. Bauml J, Pitschel-Walz G, Volz A, Engel RR, Kessling W. Psychoeducation in Schizophrenia: 7-year follow-up concerning rehospitalization and days in hospital in the Munich Psychosis Information Project Study. J Clin Psychiatry 2007; 68(6): 854-61.1.

3. Magliano L, Fiorillo A, Malangone C, DeRosa C, Maj M. Patient Functioning and family burden in a controlled, real-world trial of family psychoeducation for schizophrenia. Psychiatr Serv. 2006; Dec; 57(12): 1784-91.

4. Cohen AN, Glynn SM, Murray-Swank AB, Barrio C, Fischer EP, McCutcheon SJ, et al. The family forum: directions for the implementation of family psychoeducation for severe mental illness. Psychiatr Serv. 2008 Jan; 59(1): 40-8.

5. Vieta E, Rosa AR. Evolving trends in the long-term treatment of bipolar disorder. World J Biol Psychiatry 2007; 8(1): 4-11.

6. Murray-Swank AB, Dixon L. Family psychoeducation as an evidence-based practice. CNS Spectr. 2004; Dec; 9(12): 905-12.

7. Okello ES, Abbo C, Musisi S, Tusaba C. Incorporating Traditional Healers in Primary Mental Health Care in Uganda. Makerere University Research Journal 2006; 1 (2): 139-48.

8. Agara AJ, Onibi OE. Effects of Group Psychoeducation (GPE) on Compliance with Scheduled Clinic Appointments in a NeuroPsychiatric Hospital in Southwest Nigeria: A Randomised Control Trial (RCT). Ann Acad Med Singapore 2007; 36:272-6.

9. Kronmueller, K-T, Saha R, Kratz B, Karr M, Hunt A, Mundt C, Backenstrass M. Reliability and Validity of the Knowledge about Depression and Mania Inventory. Psychopathology 2008; 41: 69-76.

10. Seedat S, Haskis A, Stein DJ. Benefits of consumer psychoeducation: a pilot program in South Africa. Int J Psychiatry Med 2008; 38(1): 31-42.

11. Chien W-T, Chan SWC, Thompson DR. Effects of a mutual support group for families of Chinese people with schizophrenia: 18month follow-up. British Journal of Psychiatry 2006; 189: 41-49.

12. Bustillo JR, Lauriello J, Horan WP. The Psychosocial Treatment of schizophrenia: an update. American Journal of Psychiatry 2001; 158: 163-175.

13. Zhang $M$, Wang $M$ Li J A randomised-control trial of family intervention for 78 first-episode male schizophrenic patients: an 18-month study in Suzhou, Jiangsu. British Journal of Psychiatry 1994; 165 (suppl, 24): 96-102. 